MedienPädagogik

www. medienpaed.com
Zeitschrift für

Theorie und Praxis

der Medienbildung

ISSN 1424-3636

Themenheft Nr. 15/16: Computerspiele und Videogames

in formellen und informellen Bildungskontexten

\title{
Der gespielte Ernst des Lebens: Bestandsaufnahme und Potenziale von Serious Games (for Health)
}

\author{
Claudia Lampert, Christiane Schwinge und Daniel Tolks
}

\begin{abstract}
Serious Games werden aktuell im Zusammenhang mit den positiven Potenzialen von Computerspielen in verschiedenen Kontexten diskutiert. Im vorliegenden Beitrag werden die Entwicklung und der Forschungsstand des relativ neuen Konzeptes skizziert und gegenüber anderen Lernkonzepten wie Edutainment, Digital Game-Based Learning und E-learning abgegrenzt. Das Spektrum von Serious Games wird am Beispiel von zwei Spielen aus dem Gesundheitsbereich (Re-Mission und Fatworld) dargestellt und die Potenziale und Grenzen von Serious Games (for Health) erörtert. Da bislang nur wenige empirische Befunde zur Wirkung von Serious Games vorliegen, plädieren die Autoren für eine umfassende Grundlagenund Wirkungsforschung.
\end{abstract}

\section{Einleitung}

Computerspiele sind ein Thema, das in der öffentlichen Diskussion immer wieder und zumeist unter negativen Vorzeichen diskutiert wird. Nach der Debatte über die Auswirkungen der «Killerspiele» wird aktuell insbesondere das Suchtpotenzial von Computerspielen (z. B. Misek-Schneider 2008) thematisiert sowie der Zusammenhang zwischen Übergewicht und regelmässiger Computerspielnutzung (z. B. Vandewater et al. 2004). Im Gegenzug dazu zeichnet sich seit einiger Zeit eine Perspektive ab, die nicht zuletzt auch von den Computerherstellern forciert wird: Um dem vermeintlich schädigenden Wirkungen von Computerspielen etwas entgegen zu setzen, wird der Blick verstärkt auch auf die ebenso vermeintlich positiven Potenziale gelenkt, die insbesondere in der Gesundheitsförderung und im Bildungsbereich gesehen werden. Beispiele sind etwa die sogenannten Exergames (Exercise + Games), wie sie vor allem für die Wii (Nintendo) und für EyeToy (Sony) angeboten werden, bei denen man Körpereinsatz zeigen muss, um einen Spielerfolg zu erzielen, Dr. Kawashimas Gehirnjogging (Nintendo), um die geistige Fitness zu trainieren sowie sogenannte Serious Games, die als unterhaltsame, interaktive Bildungsprogramme betrachtet werden. Die letztere Gruppe von Spielen steht im Mittelpunkt des vorliegenden Beitrags. Damit wird eine Kategorie in den Blick genommen, die in Deutschland noch vergleichsweise wenig berücksichtigt und untersucht wurde, aktuell jedoch - nicht zuletzt aus Marketinggründen - zu- 
nehmend an Bedeutung gewinnt. Laut van Eck (2006) belief sich der Umsatz von Serious Games im Jahre 2006 auf 20 Millionen US-Dollar. Die zunehmende Bedeutung und das wachsende Interesse an dem Bereich von Serious Games zeigen sich ebenso an der steigenden Zahl von Angeboten von Serious Games sowie im akademischen Bereich durch die zunehmende Zahl von Forschungsvorhaben, Veranstaltungen und Veröffentlichungen. Dabei handelt es sich bei den Serious Games keineswegs um ein neues Phänomen, ihr Potenzial wird bereits seit den 1970er Jahren diskutiert (vgl. Abt 1971).

Im Folgenden werden zentrale Entwicklungslinien skizziert und ein Versuch einer Eingrenzung des Themenfeldes unternommen, indem Serious Games von anderen Formen computerbasierten spielerischen Lernens abgegrenzt werden. Am Beispiel des Themenfeldes Gesundheit wird diese Spielekategorie illustriert und zugleich wird auf der Basis vorliegender Studien ausgelotet, welche Bildungspotenziale Serious Games bieten.

\section{Entwicklungslinien der Serious Games}

Die Entwicklung von Serious Games blickt auf eine sehr junge Geschichte zurück. Der Begriff Serious Games wurde erstmals 1970 von Clark C. Abt in seinem Buch Serious Games erwähnt, in dem er beschreibt, wie Spiele ${ }^{1}$ als Simulationen genutzt und in der Bildung eingesetzt werden können, aber erst 2002 wurde die Spielegattung und -bezeichnung Serious Games durch die Veröffentlichung des Spiels America's Army ${ }^{2}$ durch die US-Armee geprägt. Beinahe zeitgleich veröffentlichte Ben Sawyer ein White Paper zum Thema Serious Games und gründete mit David Rejeski die Serious Games Initiative im Woodrow Wilson International Center in Washington D.C., wodurch der Begriff Serious Games - zumindest im englischsprachigen Raum - fest etabliert wurde. Seitdem wurden zum einen weitere Institutionen gegründet, die sich mit Serious Games beschäftigen, zum anderen wurden bei bereits bestehenden Institutionen Arbeits- und Forschungsbereiche zu Serious Games ausdifferenziert. ${ }^{3}$ Ebenso ist ein stetiger Anstieg von Internetseiten und Veranstaltungen zu verzeichnen, die sich dieser Spielegattung widmen. ${ }^{4} \mathrm{Ne}-$ ben den USA und Japan werden seit 2007 vermehrt auch im europäischen Raum Forschungszentren ${ }^{5}$, Veranstaltungen und Forschungsvorhaben durchgeführt so-

1 Der Autor bezieht sich in seinem Buch allerdings schwerpunktmässig auf herkömmliche, nicht-digitale Spiele.

2 Bei dem Online-Spiel America's Army handelt es sich um einen kostenlosen First-Person-Shooter, der als Rekrutierungswerkzeug der US-Armee konzipiert wurde. Seit der Einführung des Spieles konnte ein Anstieg der Rekrutierungszahlen der US-Armee verzeichnet werden.

3 Serious Games Institute, Liemand Foundation, MIT Comparative Media Studies, CMP Game Group, Robert Wood Johnson Foundation.

4 Siehe hierzu z. B. die Serious Games Summits der CMG Game Group und der First Educaton Arcade: Games in Education der MIT Comparative Media Studies.

5 Beispiele sind: das Serious Games Institute, die Digital Games Research Association (DiGRA) oder das Center for Advanced Media Research Amsterdam (CAMeRA). 
wie eigene Serious Games konzipiert. In Deutschland wurde 2007 zum ersten Mal die Serious Games Conference im Rahmen der CeBit veranstaltet und der Serious Games Award des hessischen Wirtschaftsministeriums vergeben. Unter den Experten im Bereich Serious Games besteht kein Zweifel, dass sich dieses Feld kontinuierlich weiterentwickeln wird (Susi et al. 2007, Sawyer 2005; 2007, van Eck 2006).

Serious Games sind - wie andere Spielegattungen auch - thematisch nicht festgelegt. Laut Ritterfeld (2008)6 setzen sich 63 Prozent der Spiele mit Themen im schulischen Kontext auseinander, 14 Prozent mit gesellschaftlich relevanten Themen, 10 Prozent der Spiele werden zur beruflichen Weiterbildung eingesetzt, 8 Prozent der Spiele behandeln die Thematik Medizin und Gesundheit, 5 Prozent werden zu militärischen Zwecken eingesetzt und ca. ein Prozent entfallen auf Marketing Games. Dabei werden Serious Games für alle Computerspielgenres entwickelt, wie in den Bereichen Simulationen (z. B. Pulse), First-Person-Shooter (z. B. ReMission), Rollenspiele (z.B. Escape from Diab), Casual- und Arcade-Games (z.B. Catch the Sperm, Feed the Monster), Adventures (z. B. Heart Sense, Outbreak at Water's Edge), Strategiespiele (z. B. Global Conflicts: Palestine, PeaceMaker) und Wirtschaftssimulationen (z. B. Hungry Red Planet).

Uneinigkeit besteht allerdings hinsichtlich der Definition des Begriffes «Serious Games». Dies zeigt sich u. a. auch an der Vielzahl von existierenden Begriffsbestimmungen, von denen sich bislang keine als einheitliche Definition durchsetzen konnte. So werden die Termini Serious Games, Educational Games, Edutainment, Digital Game-Based Learning, Social Impact Games, Persuasive Games, Games for Change bisweilen synonym verwendet (vgl. Susi et al. 2007, Sawyer und Smith 2008). In der ersten Definition zu Serious Games von Abt (1971) wird ein explizit formuliertes Bildungsziel als zentrales Abgrenzungsmerkmal von Serious Games gegenüber anderen Spielen betont:

Wir haben es hier mit ernsten Spielen in dem Sinne zu tun, dass diese Spiele einen ausdrücklichen und sorgfältig durchdachten Bildungszweck verfolgen und nicht in erster Linie zur Unterhaltung gedacht sind. Das heisst nicht, dass ernste Spiele nicht unterhaltsam sind oder sein sollten. (Abt 1971: 26, Hervorheb. i. Orginal)

Die Definition der Serious Games Initiative verweist ebenfalls auf die pädagogische Intention von Serious Games, unterstreicht jedoch noch die «Ernsthaftigkeit» der Spiele, indem sie die gesellschaftliche und gesundheitliche Relevanz der Spiele betont (siehe auch Zyda 2005).

6 Ritterfeld bezieht sich dabei auf Ergebnisse einer von ihr durchgeführten Studie, die Ende 2008 als Buch mit dem Titel: «Serious Games: Mechanisms and Effects» veröffentlicht werden sollen. 
The Serious Games Initiative is focused on uses for games in exploring management and leadership challenges facing the public sector. Part of its overall charter is to help forge productive links between the electronic game industry and projects involving the use of games in education, training, health, and public policy. (Quelle: Serious Games Initiative: http://www.seriousgames. org/about2, 29.7.08)

Wie bei allen Entertainment-Education-Angeboten (vgl. u.a. Singhal \& Rogers 1999, Singhal et al. 2004, Lampert 2007) stellt sich auch für die Serious Games die Frage, in welchem Verhältnis der Bildungs- und Unterhaltungsaspekt stehen. Einigen Autoren zufolge ist ein Hauptmerkmal von Serious Games, dass der Unterhaltungscharakter dem Bildungsanspruch nachgeordnet ist; entsprechend handelt es sich um «[...] games that do not have entertainment, enjoyment, or fun as their primary purpose» (Michael \& Chen 2006: 4). Andere verweisen darauf, dass sich der pädagogische Anteil der Entertainment-Komponente unterordnen muss (vgl. Zyda 2005). Ein Konsens konnte bislang nur dahingehend erzielt werden, dass es sich bei Serious Games um Computerspiele handelt, die nicht allein auf Unterhaltung abzielen, sondern darüber hinaus andere Inhalte vermittelt sollen. Dabei ist keinesfalls festgelegt, um welche Art von Inhalten es sich handelt, und Beispiele wie America's Army verweisen darauf, dass sich Serious Games auf einem sehr schmalen Grat zwischen Bildungsangebot und Propagandamittel bewegen. Momentan wird der Begriff für alle Computerspiele benutzt, die für Simulationen, Bildung und Trainingszwecke in unterschiedlichen Anwendungsgebieten (z. B. Bildungssektor, Medizin, Militär) eingesetzt werden, was eine klare Abgrenzung zu anderen Computerspielen erschwert, die eine pädagogische Intention verfolgen. Verschiedene Autoren vertreten die Auffassung, dass es auch in nächster Zeit keine einheitliche Definition geben wird (vgl. Susi et al. 2007, Sawyer 2007). Gründe dafür werden vor allem in den unterschiedlichen Anwendungsgebieten und Interessengruppen gesehen. Für z. B. die Computerspieleindustrie, die aktuell ein Interesse daran hat, ihre Produkte mit der Kennzeichnung Serious Games zu vertreiben, wäre - zumindest zu diesem Zeitpunkt - eine zu starke Eingrenzung des Feldes für die weitere Entwicklung dieses Marktsegmentes eher hinderlich.

\section{Bildende Spiele oder spielerische Bildung? Abgrenzung zu anderen Konzepten}

Neben den Serious Games gibt es eine ganze Reihe von Ansätzen und Konzepten, die sich das Unterhaltungspotenzial von Computerspielen für die Vermittlung pädagogischer Inhalte zunutze machen wie z. B. E-learning, Edutainment und (Digital) Game-Based Learning (vgl. auch Vollbrecht 2008). Um die Serious Games im Feld der pädagogisch motivierten Computerspiele zu verorten, werden die anderen Variationen im Folgenden kurz skizziert. Teilweise sind die Grenzen zwischen den 
verschiedenen Angeboten hinsichtlich ihrer pädagogischen Intention (bewusst) fliessend und die unterschiedlichen Angebotskategorien nicht völlig trennscharf: «Übergänge vom intentionalen, gezielten Lernen zum incidentellen, unsystematischen, beiläufigen Lernen sind ständig gegeben und werden heute zudem von vielen modernden Medien geradezu forciert» (Kübler 1997: 41). Entsprechend sind an verschiedenen Punkten Überschneidungen mit der Kategorie Serious Games erkennbar bzw. eine klare Abgrenzung nicht möglich.

Die umfassendste Kategorie stellt sicherlich das E-learning dar, das sich laut Hodson et al. (2001) vor allem auf Aspekte des computerbasierten Lernens, auf interaktive Technologien und weitergehend auf das Lernen auf Distanz bezieht (zit. n. Susi et al. 2007, 2). Edutainment', ein Hybrid aus Education und Entertainment, das vor allem in den 1990er Jahren geprägt wurde, bezieht sich - wie Serious Games auch - auf Bildungsangebote bzw. spielerische multimediale Lernumgebungen, die ebenfalls unterhalten sollen bzw. auf «anspruchsvolle» Unterhaltungsangebote, die gleichzeitig bilden (vgl. Aufenanger 2005). ${ }^{8}$ Der Fokus liegt vor allem auf der Vermittlung von Themen im schulischen Kontext, wie z. B. Sprachen, Mathematik, Physik oder Chemie. Serious Games verfolgen grundsätzlich das gleiche Ziel, gehen aber weit über den Aspekt der reinen Wissensvermittlung hinaus und zielen auf Bildung allgemein oder Training (vgl. Michael \& Cheng 2006). Ebenso beschränkt sich die Zielgruppe nicht auf Kinder, sondern umfasst alle Altersgruppen. Eine ähnliche Abgrenzung nimmt auch Egenfeldt-Nielsen (2005) vor. Auch er sieht den Fokus bei Edutainment-Angeboten auf dem Trainieren von Fähigkeiten bzw. basalen Kompetenzen, über die man bereits verfügt, z. B. rechnen, lesen, schreiben. Bei Serious Games hingegen ist aus seiner Sicht das Lernen neuer Fähigkeiten und Kompetenzen zentral. Während bei Edutainment-Titeln in der Regel der Spielteil als Belohnung für das Gelernte erfolgt, also nicht integrativer Bestandteil dessen ist, was man lernt, werden bei Serious Games die Lerninhalte und -aufgaben («educational missions) in die Spielwelt integriert (vgl. ebd.). Im Gegensatz zu Edutainment-Titeln, bei denen aufgrund einer starken Lernkomponente oftmals eine fehlende motivierende Dynamik mit einhergeht, verfügen Serious Games über Eigenschaften, die für kommerzielle Computerspiele charakteristisch sind, wie z. B. der hohe Motivationsgrad oder ausdifferenzierte Spielumgebungen (vgl. Egenfeldt-Nielsen 2006).

Game-Based Learning und Digital Game-Based Learning sind weitere Konzepte, die Spiele für pädagogische Zwecke nutzen, wobei Digital Game-Based Learning das Handlungsfeld auf digitale Spiele begrenzt. Prensky (2001a) definiert diese Form spielerischen Lernens als «[...] any learning game on a computer or online».

7 Auch: education through entertainment.

81984 wurde das Label Edutainment erstmals für ein Spiel vergeben. Das Adventure/Arcade Videogame The Seven Cities of Gold wurde von Electronic Arts als Entertainmenttitel vermarktet (Egenfeldt-Nielsen 2006: 184). 
Darüber hinaus muss ein Angebot dieser Kategorie verschiedene Eigenschaften aufweisen, z. B. müssen Kontext und Inhalt so miteinander verbunden sein, dass sich der Nutzer des Spiels die ganze Zeit wie ein Spieler fühlt und nicht wie ein Lernender. Ausserdem funktioniere Digital Game-Based Learning nur dann, wenn Engagement und Lernen gleich (hoch) gewichtet sind. Ist dies nicht der Fall, wird das Spiel entweder zum Lernprogramm, oder aber zu einem gewöhnlichen (Unterhaltungs-) Computerspiel. Zudem müssen die beiden Dimensionen während des kompletten Spielablaufs auf einem hohen Niveau gehalten werden. Digital GameBased Learning funktioniere vor allem aufgrund der spielinhärenten Motivation, die den Spieler unbewusst zum Lernen bringe (stealth learning). Hinsichtlich der Zielgruppen und Anwendungsbereiche sieht Prensky keine Einschränkungen. Einsatzbereiche sind u.a. unterschiedliche formale Bildungsbereiche, Betriebe, Militär oder Organisationen.

Den Gegenpol zu den pädagogisch motivierten Spielen bilden die sogenannten Entertainment Games, d.h. allgemeine kommerzielle Computerspiele. ${ }^{9}$ Diese Spiele beinhalten bisweilen Elemente, die den Spieler ins Spiel eintauchen lassen, also zu einem immersiven Erlebnis führen. Im Gegensatz zu Serious Games werden Entertainment Spiele in der Regel primär zu Unterhaltungszwecken gespielt, was allerdings nicht ausschliesst, dass auch hier Lernprozesse stattfinden, allerdings auf einer informellen Ebene. Darüber hinaus ist selbstverständlich auch vorstellbar, dass Entertainment Games unter didaktischen Gesichtspunkten in formalen Bildungskontexten eingesetzt werden, wenngleich eine zu starke Didaktisierung sich auch negativ auf den Lernerfolg auswirken kann, wenn das Spiel nicht mehr als Spiel im Hinblick auf sein Motivationspotenzial funktioniert (vgl. Vollbrecht 2008).

\section{Gesundheitsförderung durch Computerspiele: Serious Games for Health}

Die Potenziale von Serious Games werden aktuell vor allem im Zusammenhang mit medizinischen bzw. der Vermittlung von gesundheitsbezogenen Themen diskutiert, weshalb im Folgenden der Fokus auf die Unterkategorie Serious Games for Health gerichtet wird. 2004 wurde mit dem Games for Health Project das erste Institut im Feld Computerspiele und Gesundheit gegründet..$^{10} 2007$ folgte die Einrichtung des Health Games Research Program an der University of California Santa Barbara, welches den Forschungsbereich Computerspiele und Gesundheit erweitern soll. ${ }^{11}$ In Deutschland zeichnet sich ebenfalls zunehmend ein Interesse an dieser Thematik ab: Im März 2008 veranstalteten der Bundesverband Interaktive Un-

9 Auch COTS Games - Commercial Off The Shelf Games genannt.

10 Das Games for Health Project veranstaltet jährlich die Games for Health Conference, die als Plattform für den Austausch unter den Akteuren im Bereich Serious Games for Health dient.

11 Insgesamt 8,25 Millionen US-Dollar investierte die Robert Wood Johnson Foundation in dieses Programm. 
terhaltungssoftware e.V. und die nordmedia (Mediengesellschaft Niedersachsen/ Bremen mbH) im Rahmen der CeBit eine Konferenz zum Thema "Serious Games for Health - Spiele in der Medizin».12

Bei den Serious Games for Health handelt es sich nicht um Computerspiele, die im eigentlichen Sinne als Unterhaltungsmedium konzipiert wurden und für gesundheitsförderliche Zwecke eingesetzt werden, wie z. B. Wii-Sports in der Rehabilitation, sondern um Computerspiele, die speziell entwickelt werden, um auf den Gesundheitszustand des Nutzers gezielt positiv einzuwirken. Laut Ritterfeld (2008) beschäftigen sich 8 Prozent aller Serious Games mit dem Thema Medizin und Gesundheit. Auch in diesem Feld gibt es eine Vielzahl an Angeboten, die sich auf unterschiedliche Bereiche konzentrieren ${ }^{13}$. So werden Serious Games in verschiedenen medizinischen Zusammenhängen eingesetzt, z. B. bei Schulungen von medizinischem Personal, als therapeutisches Element bei psychischen und physischen Erkrankungen oder - wenngleich eher selten - im Bereich Prävention und Gesundheitsförderung (z. B. als Aufklärungsmittel). Behandelte Themen erstrecken sich bislang von Erkrankungen HIV/AIDS, Asthma bronchiale, Diabetes mellitus bis hin zu psychischen Erkrankungen wie z. B. Schizophrenie und Phobien.

Im Folgenden wird die Breite des Angebots an Serious Games for Health am Beispiel von zwei ausgewählten Spielen illustriert. Bei dem ersten Spiel Re-Mission handelt es um eines der prominentesten Beispiele, das im therapeutischen Bereich eingesetzt wird, während das zweite Beispiel Fatworld dem Bereich Prävention und Gesundheitsförderung zuzuordnen ist.

Beispiel 1: Re-Mission

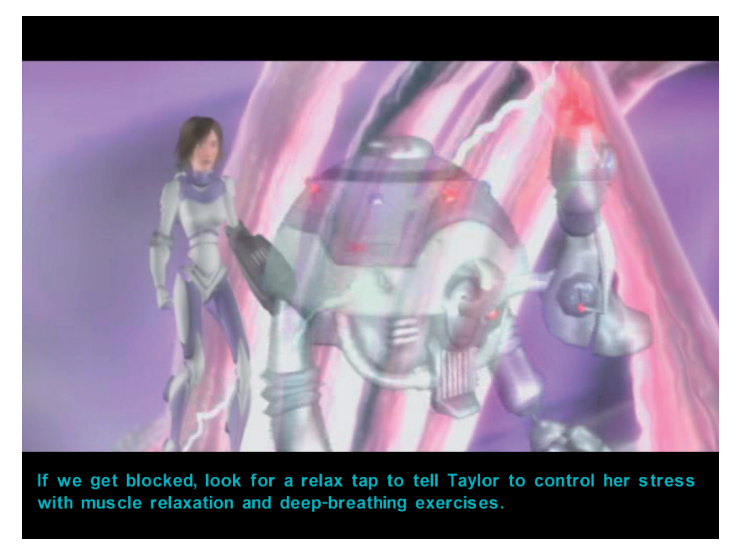

Abb. 1: Cutscene aus Re-Mission (@ Realtime Associates)

12 http://www.seriousgames-conference.de/index.php?id=37\&tx_ttnews[tt_news]=3\&tx_ttnews[backP id] $=1 \& \mathrm{cHash}=061290 \mathrm{da} 33$.

13 Eine umfassende Übersicht sowie kurze Beschreibung von über 500 Serious Games wird auf der Internetseite von Marc Prensky http://www.socialimpactgames.com dargestellt. 
Bei Re-Mission handelt es sich um das derzeit wohl bekannteste und am besten evaluierte Serious Game im Bereich Gesundheit ${ }^{14}$. Entwickelt wurde das Spiel mit einem, im Vergleich zu anderen Serious-Games-Produktionen, hohen Budget von 4,5 Millionen US-Dollar von Realtime Associates im Auftrag der Non-ProfitOrganisation HopeLab. Ziel von HopeLab ist die Verbindung von umfassender Forschung mit innovativen Lösungen, um die Gesundheit und Lebensqualität junger Menschen mit chronischen Krankheiten zu verbessern. Zusammen mit Onkologen, Psychologen und Videospiel-Designern wurde ein Computerspiel für Kinder und Jugendliche mit bösartigen Tumorerkrankungen entwickelt. Ziel des Spiels ist es, das Verhalten und die Einstellung der jungen Patienten bezüglich ihrer Erkrankung positiv zu beeinflussen. In dem Spiel werden in zwanzig Levels sieben verschiedene Krebserkrankungen und ihre Nebenwirkungen thematisiert. Wie in einem First-Person- bzw. Ego-Shooter fliegt der Spieler mit seiner Spielfigur, dem Nanoroboter Roxxi, durch den Körper von virtuellen Patienten, um gegen Tumorzellen und weitere Nebenwirkungen von Krebserkrankungen zu kämpfen. Dabei können unterschiedliche Waffen genutzt werden, die mit Chemotherapiepräparaten, Antibiotika und anderen Medikamenten geladen werden und mit dem der Spieler auf die Tumorzellen und Bakterien schiesst (vgl. Abb. 1). Eine Vielzahl von «Cutscenes» ${ }^{15}$ wird genutzt, um weitere gesundheitsrelevante Informationen zu vermitteln (vgl. Abb. 1). Bestandteil des Gameplays ist auch die Kommunikation mit dem Patienten. Zum Beispiel kommuniziert die Spielfigur mit dem virtuellen Patienten während einer Untersuchung und weist ihn an, sich zu entspannen um etwaige Blockaden zu lösen (vgl. Abb. 2). Daraufhin wird der weitere Weg in dem Level freigeschaltet.

Bislang wurden 100.000 Exemplare in drei Sprachen (Englisch, Spanisch und Französisch) in 78 Ländern verteilt. Darüber hinaus hat sich auf der Re-Mission-Website

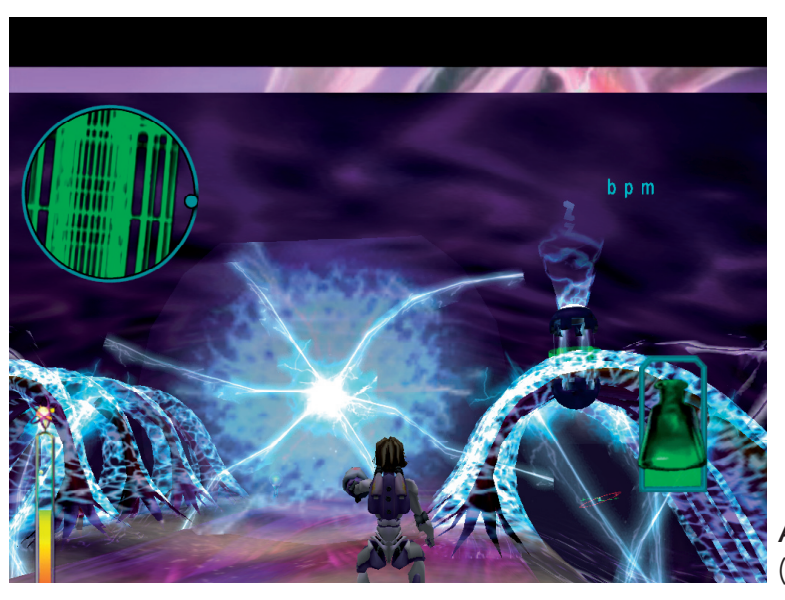

Abb. 2: Screenshot aus Re-Mission (C) Realtime Associates)

14 Re-Mission kann man auf www.re-mission.net downloaden. Auf dieser Seite gibt es zudem umfangreiche Informationen und ein Forum.

15 Bei Cutscenes handelt es sich um kurze Einspielfilme während des Computerspiels. 
eine aktive Community gegründet, in der sich die betroffenen Kinder und Jugendlichen über ihre Krankheit austauschen können. Hierfür wurden Foren eingerichtet und es besteht die Möglichkeit der Erstellung eigener themenbezogener Blogs, mit dem Ziel die Diskussion in peer-groups zu fördern. Weiterhin können neben den krebsrelevanten Themen auch eigene Gedichte und Bilder präsentiert werden. Ebenso wird die Möglichkeit gegeben, sich über die spezifischen Erkrankungen zu informieren und direkte Fragen an das Team von Re-Mission und HopeLab zu stellen. Weiterführende krankheitsrelevante Links werden auf der Website zur Verfügung gestellt.

\section{Beispiel 2: Fatworld}

Bei Fatworld handelt es sich um ein Serious Game, das im Bereich Prävention und Gesundheitsförderung eingesetzt wird. Entwickelt wurde es von Persuasive Games,

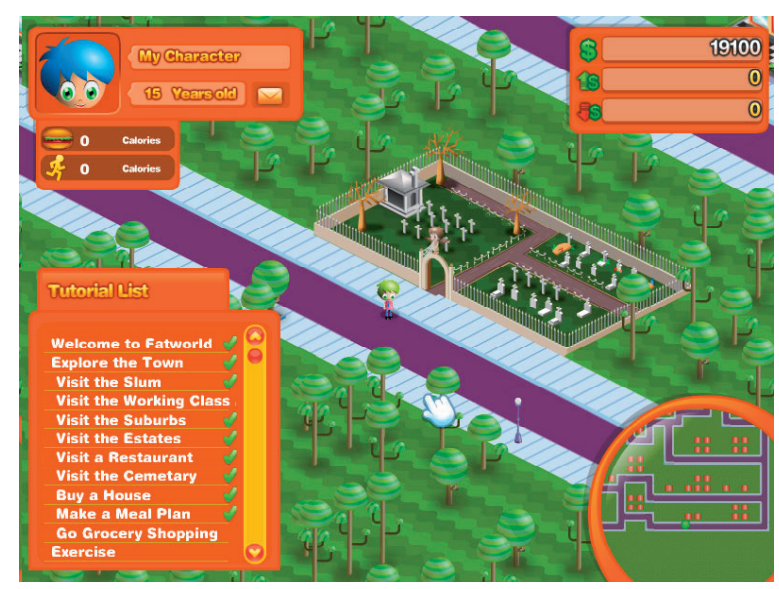

Abb. 3: Szene aus dem Spiel Fatworld (@ Persuasive Games)

finanziert von der Corporation for Public Broadcasting (CPB). Das Spiel wurde im Januar 2008 veröffentlicht und steht zum kostenlosen Download zur Verfügung. ${ }^{16}$ Ziel des Spieles ist es, die Zusammenhänge der Bedingungsfaktoren (Determinanten) von Übergewicht darzustellen. Die Auswirkungen eines gesundheitsförderlichen oder auch eines ungesunden Lebensstils sollen im Zeitraffer veranschaulicht werden. Dem Spieler sollen so die längerfristigen Auswirkungen des Lebensstils auf die Gesundheit aufgezeigt werden. Zu diesem Zweck wird eine virtuelle Stadt simuliert, durch die die Spielfigur gesteuert wird. Bei Fatworld handelt es sich um ein sogenanntes "Sandbox Game»" ${ }^{17}$, das sich an populären Computerspielen wie

16 Unter www.fatworld.org ist das Spiel erhältlich. In den ersten zwei Wochen nach der Veröffentlichung wurde Fatworld bereits 53.000 mal heruntergeladen.

17 Bei einem «Sandbox Game» wird kein klares Spielziel oder ein definiertes Ende vorgegeben. Dem Spieler wird die Möglichkeit gegeben, eigene Ziele zu definieren und den Spielverlauf durch eigene Entscheidungen zu verändern. 
Die Sims oder der GTA-Serie orientiert. Der Spieler kann zu Beginn seine Spielfigur individuell gestalten. Dazu gehören neben Erscheinungsbild und Gewicht auch die Auswahl von Dispositionen von chronischen Erkrankungen, die durch Übergewicht bedingt werden, sowie die Wahl des sozioökonomischen Status. Der Spieler navigiert die Spielfigur durch eine virtuelle Stadt und hat die Möglichkeit, unterschiedlichste Aktivitäten durchzuführen, die sich auf den Gesundheitszustand der Spielfigur sowie auf die Bewohner der Stadt auswirken (vgl. Abb. 3). Dazu gehören die Erstellung eigener Rezepte und Mahlzeiten sowie das Einkaufen der benötigten Lebensmittel. Der Spieler hat die Möglichkeit, ein Restaurant zu betreiben und durch die Menüwahl die Gesundheit der anderen Bewohner zu beeinflussen. Ausserdem besteht die Möglichkeit, seinen eigenen Gesundheitsstatus in einem sogenannten Health-O-Mat darstellen zu lassen. Auf der politischen Ebene kann der Spieler durch den Govern-O-Mat direkt auf die örtliche Politik einwirken, indem er durch die Zahlung von Geldern dafür sorgen kann, dass bestimmte Nahrungsmittel oder Inhaltsstoffe in der virtuellen Stadt nicht mehr verkauft bzw. verstärkt angeboten werden, oder die Kosten für Gesundheitsdienstleistungen reduziert werden. Fatworld simuliert eine persistente Spielwelt, die sich also auch während der Zeit verändert, in der der Nutzer nicht spielt. Auf der Internetseite von Fatworld werden Informationen und weiterführende Links zum Thema Übergewicht angeboten.

Re-Mission und Fatworld sind nur zwei Beispiele, die das breite Spektrum von Serious Games mit Gesundheitsbezug aufzeigen. Vor allem am Beispiel von Fatworld lassen sich einige Gründe für die steigende Anzahl von Spielen mit dem Label Serious Games und gleichzeitig die Problematik dieser Labelung verdeutlichen. Die Fachpresse charakterisierte Fatworld im Vorfeld der Entwicklung als ein Spiel, das ein hochrelevantes Thema behandelt und sich mit innovativen Ideen dieser Thematik annimmt. Nach der Veröffentlichung wurde in der Fachpresse weiterhin auf die Wichtigkeit der Thematik des Computerspieles hingewiesen, ohne auf die Spielqualität an sich einzugehen. Die eigentliche niedrige Qualität des Computerspieles wurde allenfalls von privaten Nutzern in Blogs thematisiert. Bei Fatworld wurde demzufolge das Label Serious Games genutzt, um die Popularität des Spieles zu erhöhen und gleichzeitig die Erwartungshaltung an die Qualität zu verringern.

\section{Zu Potenzialen und erwünschten Nebenwirkungen von Serious Games}

Bei pädagogisch motivierten Angeboten stellt sich grundsätzlich die Frage nach ihrem Erfolg, d.h., ob die ursprüngliche Intention erreicht werden konnte. Die Operationalisierung der Kategorie Erfolg, aber auch die vielfältigen Themen, Intentionen und Umsetzungsmöglichkeiten erschweren eine eindeutige Antwort. Für Serious Games allgemein und insbesondere denen mit Gesundheitsbezug liegen bislang nur wenige Studien vor, die einen tatsächlichen Effekt - in diesem 
konkreten Fall auf den Gesundheitszustand der Spieler - nachweisen konnten. Erste Studien zum Einsatz von Computerspielen im Bereich Disease Management wurden bereits Ende der 1990er Jahre durchgeführt, ohne dass das Label Serious Game verwendet wurde. Dabei wurden Video-Spiele, die für Kinder mit einer Diabetes-Mellitus-Erkrankung (Packy \& Marlon) oder Asthma bronchiale (Bronkie the Bronchiasaurus) entwickelt wurden, dahingehend untersucht, ob sie das krankheitsbezogene Selbstmanagement von Kinder verbessern konnten. In den Untersuchungen zum Spiel Packy \& Marlon konnten signifikante Verbesserungen des krankheitsbezogenen Selbstmanagements (regelmässige Messung des Blutzuckerspiegels, adäquate Substitution von Insulin, Einhaltung der Diätvorschriften) und der krankheitsbezogenen Selbstwirksamkeitserwartung verzeichnet werden (Brown et al. 1997, Lieberman 1998). Die Zahl der krankheitsbezogenen Notaufnahmen konnte um 77 Prozent reduziert werden (ebd.). Die Wirksamkeit des Spieles Bronkie the Bronchiasaurus wurde in drei Studien untersucht. Die Befunde zeigten eine signifikante Verbesserung der Einstellung, des Verhaltens und des Gesundheitszustandes bezüglich der asthmatischen Erkrankung der Probanden (Lieberman 2001). Zudem konnte eine Steigerung der Selbstwirksamkeitserwartung, ein Wissenszuwachs und eine Verbesserung der Selbstfürsorge bezüglich der Erkrankung festgestellt werden (ebd.).

Speziell zur Spielegattung Serious Games for Health und ihrer Wirksamkeit gibt es bislang nur wenige Studien, was u. a. darauf zurückzuführen ist, dass der Gegenstandsbereich bzw. die Spielegattung (noch) nicht eindeutig eingegrenzt werden kann (vgl. Kap. 2). Im Rahmen einer Übersichtsstudie der vorliegenden Forschungsliteratur wurde festgestellt, dass bei 24 von 25 Serious Games, die im Medizin- oder Gesundheitsbereich eingesetzt wurden, Indikatoren für eine Wirksamkeit, wie z. B. Wissenszuwachs, Verhaltens- und/oder Einstellungsänderungen, vorlagen (vgl. Baranowski et al. 2008).

Auch mit Blick auf einzelne, eindeutig als Serious Games definierte Spiele, lässt sich die empirische Datenlage als sehr dünn bezeichnen. Nur vereinzelt wurden zu Spielen, wie z.B. zum oben skizzierten Spiel Re-Mission, aufwändige Evaluationsstudien durchgeführt, die in eine positive Richtung weisen. Bereits vor der offiziellen Veröffentlichung wurde Re-Mission auf seine Wirksamkeit auf der Nutzerebene überprüft. Die Studie wurde von der Stanford University mit 375 Krebspatienten zwischen 13 und 19 Jahren in 34 medizinischen Zentren und Krankenhäusern in den USA durchgeführt. Untersucht wurden die generelle Compliance, die subjektive Einschätzung der Lebensqualität, die krankheitsbezogene Selbstwirksamkeitserwartung sowie der krankheitsbezogene Wissenszuwachs. Im Zuge der Studie konnte bei den untersuchten Personen eine signifikant höhere Konzentration von krankheitsrelevanten Medikamenten und Chemotherapiepräparaten nachgewiesen werden (Kato et al. 2006). Des Weiteren wurde eine Steigerung des krankheitsrelevanten Wissens und der krebsbezogenen Selbstwirksamkeitserwartung 
nachgewiesen (ebd.). Bei der subjektiven Einschätzung der Lebensqualität (Pediatric Quality of Life Scale) der Patienten konnte ebenso ein Anstieg verzeichnet werden.

Die wenigen vorliegenden Befunde unterstreichen zwar die Potenziale von Serious Games, sind aber nicht hinreichend, um ein abschliessendes Urteil fällen zu können. Hier sind weitere Evaluations- und Wirkungsstudien notwendig, die die Leistung der Spiele am jeweiligen Einzelfall differenziert untersuchen und weitere Faktoren (z. B. Alter und Geschlecht) einbeziehen. Die Ergebnisse der empirischen Studien deuten allerdings darauf hin, dass Serious Games insbesondere in den Bereichen geeignet sind, in denen ein enger Handlungsrahmen vorliegt: Je präziser die Zielformulierung und die Ausrichtung an der Zielgruppe ist, desto höher ist die Wirksamkeit bzw. der Lernerfolg dieser Spiele einzuschätzen (vgl. das Beispiel Re-Mission). Gerade im Hinblick auf die Bereiche Compliance und Therapie scheint daher der Einsatz von Serious Games ein viel versprechender Ansatz zu sein, um bestimmte (vor allem computerspielaffine) Zielgruppen mit gesundheitsbezogenen und -relevanten Botschaften zu erreichen.

\section{Fazit und Ausblick}

Computerspiele sind gemeinhin bei Kindern und Jugendlichen sehr beliebt und daher besonders geeignet, um die Zielgruppe der sogenannten «digital natives» (Prensky 2001b) zu erreichen. Gegenüber pädagogisch motivierten Angeboten im Fernsehen - seien es Entertainment-Education-Angebote oder reine Bildungssendungen - haben Computerspiele allgemein den Vorteil, dass sie Möglichkeiten zur Interaktion bieten, was das Gefühl der Selbstwirksamkeit auf Seiten der Spieler erhöht. Darüber hinaus sind sie hoch motivierend, passen sich an das Niveau der Spieler an und führen so zu Erfolgserlebnissen (Vollbrecht 2008, Fritz 2003). Insgesamt bieten sie also günstige Voraussetzungen, sowohl für intentionales, als auch für inzidentelles Lernen. Gegenüber Edutainment-Titeln haben Serious Games den Vorteil, dass das Gameplay im Vordergrund steht (bzw. stehen sollte), was sich positiv auf die Motivation auswirkt, sich längerfristig mit einem Spiel und entsprechend auch mit einem bestimmten Thema zu beschäftigen. Eine hohe Motivation und Involvierung der Spieler haben wiederum den Effekt, dass die im Spiel erlebten Erfahrungen bei den Spielern länger gespeichert bleiben, die Retention des Gelernten im Vergleich zu anderen Lehrformen sich also erhöht (vgl. Egenfeldt-Nielsen 2005).

Wenngleich die Potenziale von Computerspielen allgemein, aber auch von Serious Games im Besonderen für pädagogische Ziele grundsätzlich als durchaus hoch eingeschätzt werden, ist jedoch auch auf einige Grenzen hinzuweisen. So stehen zum Beispiel pädagogische Angebote im Spielebereich grundsätzlich vor der Herausforderung, einerseits niedrige Systemanforderungen zu stellen, um auch diejenigen mit ungünstigeren technischen Voraussetzungen zu erreichen, an- 
dererseits aber in Bezug auf Spielegrafik und Gameplay mit Entertainment-Titeln konkurrieren zu können. Beide Faktoren sind mit Blick auf potenzielle Nutzergruppen von Serious Games sorgfältig abzuwägen.

Serious Games sind - wie der Blick auf die anderen Varianten von spielerischern Lern- und Bildungsangeboten zeigt - von der Idee her nicht neu, sondern machen sich lediglich die Vorteile und Potenziale virtueller Spielewelten für bestimmte, meist sozialrelevante Themenbereiche zu nutzen. Der Erfolg von Serious Games wird daher, wie bei anderen pädagogischen Medienangeboten bzw. computerbasierten Bildungsangeboten auch, sehr stark von der Intention und dem Kontext abhängen, in dem ein Spiel eingebettet ist. So können konkrete Ziele in einem engen Handlungsrahmen, wie er z. B. in einem therapeutischen Kontext gegeben ist, vermutlich weitaus besser und nachhaltiger erreicht werden als Ziele im Bereich der Prävention und Gesundheitsförderung wie z. B. die Änderung von generellen Einstellungen und Verhaltensweisen.

Dies wirft wiederum die Frage nach der Ziel- und Nutzergruppe von Serious Games auf. Gänzlich neue Nutzergruppen werden sich mit diesen Angeboten kaum erschliessen lassen. Spiele wie beispielsweise Re-Mission und Fatworld werden (vermutlich) vor allem von denjenigen genutzt werden, die entweder persönlich von dem Thema betroffen oder an dem Thema interessiert sind. Angesichts des breiten Marktes an Computerspielen und der Fülle von kostenlosen Downloadspielen ist es jedoch eher unwahrscheinlich, dass sich Personen gezielt auf die Internetseiten von Serious-Games-Anbietern begeben, um sich ein sozial relevantes oder gesundheitsbezogenes Spiel herunterzuladen.

Bei gesundheitsbezogenen oder allgemein sozial relevanten Themen und Problematiken könnte es insofern eine erfolgversprechende Möglichkeit für die Zukunft sein, die zu vermittelnden Botschaften in erfolgreiche kommerzielle Computerspiele zu integrieren. Für die Bereiche Hörfunk, Film und Fernsehen finden sich inzwischen diverse Beispiele, die auf der Grundlage des Entertainment-EducationKonzepts erfolgreiche Produktionen nutzen, wie z.B. TV-Serien wie Emergency Room oder auch Gute Zeiten - Schlechte Zeiten, um bestimmte Zielgruppen mit ausgewählten Themen und Botschaften besser erreichen zu können (vgl. Lampert 2007). Im Zusammenhang mit Computerspielen bieten vor allem Onlinespiele viele Möglichkeiten, da sie - z. B. im Vergleich zu boxed products - auch nach Erscheinen leichter modifizierbar sind, aber auch bei seriellen Titeln wäre über Patches und Mods ${ }^{18}$ eine Integration von pädagogischen Botschaften durchaus denkbar. Voraussetzung ist in jedem Fall - und im Zusammenhang mit gesundheitsbezogenen Themen umso mehr - eine sorgfältige Aufbereitung der zu vermitteln-

18 Dabei handelt es sich um kleine Programme, die das Originalspiel erweitern oder verändern. Diese werden zum Grossteil von privaten Nutzern programmiert. Mods sind nur für PC-Spiele programmierbar, bei Konsolen ist eine Modifikation nicht möglich. 
den Inhalte sowie eine Form der Umsetzung, die den Unterhaltungscharakter nicht beeinträchtigt.

Die Entwicklung des Serious-Games-Marktes steht - zumindest in Deutschland noch am Anfang und es ist absehbar, dass sich das Angebot noch deutlich erweitern wird. Insbesondere im Gesundheitsbereich ist vorstellbar, dass weitere Titel entwickelt werden - vor allem solche für den Einsatz im therapeutischen Bereich, mit Blick auf eine relativ klar eingrenzbare Zielgruppe. Der Vorteil dieser Akzentuierung liegt darin, dass die Konkurrenz zu Vollpreistiteln nicht so gross ist, da durch das Gesundheits- oder Krankheitsthema ein engerer Bezug besteht, der eine höhere Involvierung und damit einen erwartbar höheren Lernerfolg begünstigt.

Für die weitere Diskussion über die Potenziale und Wirkungen dieser Spielegattung wäre eine entsprechende Begleitung dieser Entwicklung sehr wünschenswert: zum einen durch eine breitere Debatte über die Chancen und Risiken der Instrumentalisierung von medialen Unterhaltungsangeboten sowie der vertretbaren Inhalte, Werte und Normen, die über ein Spiel vermittelt werden (vgl. ebd.). Zum anderen durch systematische Bestandsaufnahmen des Angebots an (vor allem auch deutschsprachiger) Serious Games und entsprechender Nutzungsstudien, aber auch durch Evaluations- und Wirkungsstudien, um ihre Reichweite, ihren Unterhaltungswert und ihr pädagogisches Potenzial angemessen und empirisch fundiert einschätzen zu können. Eine spannende Frage, die in der öffentlichen Diskussion im Zusammenhang mit gewalthaltigen Spielen immer wieder gestellt wird und ebenso für Serious Games von Bedeutung ist, wird sein, inwieweit und unter welchen Bedingungen der Transfer vom Spiel in die Realität gelingt bzw. gelingen kann. Inhaltliche und gestalterische Aspekte werden dabei ebenso zu berücksichtigen sein, wie der lebensweltliche und spielbezogene Kontext der Spieler.

\section{Literatur}

Abt, Clark C. Ernste Spiele: Lernen durch gespielte Wirklichkeit. Köln: Kiepenheuer \& Witsch, 1971.

Aufenanger, Stefan. «Edutainment.» Grundbegriffe Medienpädagogik. Hrsg. v. Jürgen Hüther und Bernd Schorb. München: kopaed, 2005. 60-73.

Baranowski, Tom; Buday, Richard; Thompson, Debbe I.; Baranowski, Janice. «Playing for Real: Video Games and Stories for Health-Related Behavior Change.» American Journal of Preventive Medicine 34.1 (2008): 74-82.

Beale, Ivan; Kato, Pamela; Marin-Bowling, Veronica; Guthrie, Nicole; Cole, Steve W. «Improvement in cancer-related knowledge following use of a psychoeducational video game for adolescents and young adults with cancer.» Journal of Adolescent Health 41.3 (2007): 263-270.

Bopp, Matthias. Immersive Didaktik: Verdeckte Lernhilfen und Framingprozesse in Computerspielen. (2005): http://www.soz.uni-frankfurt.de/K.G/B2_2005_Bopp.pdf (29.07.2008).

Brown, S. J.; Lieberman, Debra A.; Gemeny, B.A.; Fan, Y. C.; Wilson, D. M.; Pasta, D. J. Educational video game for juvenile diabetes: Results of a controlled trial. Medical Informatics 22.1 (1997): 77-89. 
Van Eck, Richard. «Digital game-based learning: It's not just the digital actives who are restless.» EDUCAUSEreview (2006): 16-30.

Egenfeldt-Nielsen, Simon. The basic learning approach behind serious games. (2005): http:// www.seriousgames.dk/downloads/the_basic_learning_approach.pdf (20.05.2008).

Egenfeldt-Nielsen, Simon . "Overview of research on the educational use of video games.» Digital Kompetanse 3.1 (2006): 184-213.

Fritz, Jürgen. «Zwischen Frust und Flow. Vielfältige Emotionen begleiten das Spielen am Computer.» Computerspiele. Virtuelle Spiel- und Lernwelten. Hrsg. v. Jürgen Fritz und Wolfgang Fehr. Bonn: Bundeszentrale für politische Bildung, 2003: http://www.bpb.de/ themen/8GADVU,0,0,Zwischen_Frust_und_Flow.html (30.07.08).

Hodson, Peter; Connolly, Michael; Saunders, Danny. «Can computer-bases learning support adult learners?» Journal of Further and Higher Education 25.3 (2001): 325-335.

Kato, Pamela M.; Cole, Steve W.; Marin-Bowling, Veronica M.; Dahl, Gary D.; Pollock, Brad $\mathrm{H}$. Controlled trial of a video game to improve health-related outcomes among adolescents and young adults with cancer. Posterpräsentation im Rahmen der Society of Behavioral Medicine 27 ${ }^{\text {th }}$ Annual Meeting, San Francisco, USA, 2006.

Kübler, Hans-Dieter. «Bildungsmedien.» Grundbegriffe Medienpädagogik. Hrsg. v. Jürgen Hüther u. Bernd Schorb. München: KoPäd, 1997, 40-47.

Lampert, Claudia. Gesundheitsförderung im Unterhaltungsformat. Wie Jugendliche gesundheitsbezogene Botschaften in fiktionalen Fernsehangeboten wahrnehmen und bewerten. Baden Baden: Nomos Verlag, 2007

Lieberman, Debra A. "Management of chronic pediatric diseases with interactive health games: Theory and research findings.» Journal of Amulatory Care Management 24.1 (2001): 26-38.

Lieberman, Debra A. Health Education Video Games for Children and Adolescents: Theory, Design, and Research Findings. Annual meeting of the International Communication Association, Jerusalem, 1998.

Michael, David; Chen, Sande. Serious Games: Games That Educate, Train, and Inform. Boston: Thomson Course Technology, 2006.

Misek-Schneider, Karla. «Lost in Cyberspace. Können Computerspiele «süchtig» machen?» Computerspiele(r) verstehen. Zugänge zu virtuellen Spielwelten für Eltern und Pädagogen. Hrsg. v. Jürgen Fritz. Bonn: Bundeszentrale für politische Bildung, 2008. 163-183.

Prensky, Marc. Digital Game-Based Learning. St. Paul: Paragon House, 2001a.

Prensky, Marc. «Digital Natives, digital Immigrants» On the Horizon 9.5 (2001b): 1-6.

Ritterfeld, Ute. Serious games: Nur Qualität macht Spass. (2008): http://www.checkpointelearning.de/article/5425.html (12.07.08).

Ritterfeld, Ute (im Druck): Serious Games: Mechanisms and Effects. New York: Routledge

Sawyer, Ben; Smith, Peter. Serious Games Taxonomy. (2008): http://www.seriousgames.org/ index2.html (25.06.08).

Sawyer, Ben. 10 Myths about Serious Games. (2007): http://www.escapistmagazine.com/articles/view/issues/issue_121/2575-Ten-Myths-About-Serious-Games (10.06.08).

Sawyer, Ben. The State of Serious Games. (2005): http://www.gamasutra.com/features/20051024/sawyer_01.shtml (01.07.08).

Singhal, Arvind; Cody, Michael J.; Rogers, Everett M.; Sabido, Miguel (Hrsg.). Entertainment-Education and Social Change. History, Research, and Practice. Mahwah: Lawrence Erlbaum, 2004.

Singhal, Arvind; Rogers, Everett. Entertainment-Education. A Communication Strategy for Social Change. Mahwah: Lawrence Erlbaum Associates, 1999. 
Susi, Tarja; Johanneson, Mikael; Backlund, Per. Serious Games: An Overview. (2007): http:// www.his.se/upload/19354/HS-\%20IKI\%20-TR-07-001.pdf (18.06.08).

Vandewater, Elizabeth A.; Shim, Mi-suk; Caplovitz, Allison G. «Linking obesity and activity level with children's television and video game use.» Journal of Adolescence 27 (2004): 71-85.

Vollbrecht, Ralf. "Computerspiele als medienpädagogische Herausforderung.» Computerspiele(r) verstehen: Zugänge zu virtuellen Spielwelten für Eltern und Pädagogen. Hrsg. v. Jürgen Fritz. Bonn: Bundeszentrale für politische Bildung, 2008. 236-262.

Zyda, Michael. «From Visual Simulation to Virtual Reality to Games.» Computer 38.9 (2005): 25-32. 